\title{
TITLE:
}

\section{Über die Dissoziation Mehratomiger Salze}

$\operatorname{AUTHOR}(S)$ :

Sasaki, Nobuji

\section{CITATION:}

Sasaki, Nobuji. Über die Dissoziation Mehratomiger Salze. Memoirs of the College of Science, Kyoto Imperial University. Series A 1925, 7(6): 369-376

ISSUE DATE:

1925-03-30

URL:

http://hdl.handle.net/2433/256685

RIGHT: 


\title{
Über die Dissoziation Mehratomiger Salze.
}

\author{
Von
}

\section{Nobuji Sasaki.}

(Eingegangen am 17. Januar 1924)

In der vorliegenden Abhandlung soll die Abhängigkeit des Dissoziationszustandes eines mehratomigen Salzes von der Gesamt-, von der Kationen- und von der Anionenkonzentration mathematisch untersucht werden.

Als Beispiel nehmen wir Ferrichlorid, welches in folgender Schema dissoziiert :

$$
\begin{aligned}
& \mathrm{Fe}^{\cdots}+\mathrm{Cl}^{\prime} \rightleftharpoons \mathrm{FeCl}^{\circ}, \\
& \mathrm{FeCl}^{*}+\mathrm{Cl}^{\prime} \rightleftharpoons \mathrm{FeCl}_{2}, \\
& \mathrm{FeCl}_{2}{ }_{2}+\mathrm{Cl}^{\prime} \rightleftharpoons \mathrm{FeCl}_{3} .
\end{aligned}
$$

Setzt man zu dieser Lösung eine Gemenge $d^{\prime}$ von Ferrichlorid hinzu, so wird der Gleichgewichtszustand zerstört und ein neuer sich einstellen. Die Konzentrationen der Ionen $\left(\mathrm{FeCl}_{3}\right.$ einschliessend) vor und nach dem Zusatz von Ferrichlorid wollen wir durch folgende Tabelle bezeichnen :

$$
\text { Vor dem Zusatz Nach dem Zusatz }
$$

$\mathrm{Fe}^{\cdots}$
$\mathrm{FeCl}^{\circ}$
$\mathrm{FeCl}_{2}$
$\mathrm{FeCl}_{3}$
$\mathrm{Cl}^{\prime}$

$a$

$a+x$

$b \quad b+y$

$c \quad c+z$

$d \quad d+d^{\prime}-x-y-z$

$e \quad e+3 x+2 y+z$,

wobei offenbar

$$
e=3 a+2 b+c .
$$


Die Gleichgewichtsgleichungen sind demnach vor dem Zusatz

$$
\begin{aligned}
& a e=K_{1} b, \\
& b e=K_{2} c, \\
& c e=K_{3} d,
\end{aligned}
$$

und nach dem Zusatz

$$
\begin{aligned}
& (a+x)(e+3 x+2 y+z)=K_{1}(b+y), \\
& (b+y)(e+3 x+2 y+z)=K_{2}(c+z), \\
& (c+z)(e+3 x+2 y+z)=K_{3}\left(d+d^{\prime}-x-y-z\right),
\end{aligned}
$$

wodei $K_{1}$ usw. die Gleichgewichtskonstanten der einzelnen Reaktionen bezeichnen.

Falls die zugesetzte Ferrichloridmenge $d^{\prime}$ sehr klein ist, so sind $x, y$ und $z$ auch sehr klein. Die letzten drei Gleichungen können dann folgenderweise vereinfacht werden :

$$
\begin{aligned}
& \frac{x}{a}+\frac{3 x+2 y+z}{e}-\frac{y}{b}=0, \\
& \frac{y}{b}+\frac{3 x+2 y+z}{e}-\frac{z}{c}=0, \\
& \frac{z}{c}+\frac{3 x+2 y+z}{e}-\frac{d^{\prime}-x-y-z}{d}=0 .
\end{aligned}
$$

Aus diesen drei Gleichungen erhält man

$$
\begin{aligned}
& x=\frac{d^{\prime}}{d} \cdot \frac{e-2(b+c)}{b c e} \cdot \frac{1}{\Delta}=\frac{d^{\prime}}{d} \cdot \frac{3 a-c}{b c e} \cdot \frac{1}{\Delta}, \\
& y=\frac{d^{\prime}}{d} \cdot \frac{3 a+e-c}{a c e} \cdot \frac{1}{\Delta}=\frac{d^{\prime}}{d} \cdot \frac{6 a+2 b}{a c e} \cdot \frac{1}{\Delta}, \\
& z=\frac{d^{\prime}}{d} \cdot \frac{6 a+2 b+e}{a b e} \cdot \frac{1}{\Delta},
\end{aligned}
$$

wobei $\quad \Delta=\{a(b+4 c+9 d)+b(c+4 d)+c d+e(a+b+c+d)\} / a b c d e$.

Man erhält daraus noch

$$
\begin{aligned}
& 3 x+2 y+z=\frac{d^{\prime}}{d} \cdot \frac{3 a+2 b+c}{a b c} \cdot \frac{1}{\Delta}, \\
& p^{\prime}-x-y-z=\frac{d z}{c}+\frac{d^{\prime}(3 a+2 b+c)}{a b c e} \cdot \frac{1}{\Delta} .
\end{aligned}
$$


$\mathrm{Da}$, wie ersichtlich, $y ; z, 3 x+2 y+z$ und $d^{\prime}-x-y-z$ alle positiv sind, so ist es klar, dass $\mathrm{FeCl}^{*}, \mathrm{FeCl}_{2}{ }_{2}, \mathrm{FeCl}_{3}$ und $\mathrm{Cl}^{\prime}$ alle stets zunehmen müssen, wenn die Ferrichloridlösung in ihrer Konzentration zunimmt. Was $\mathrm{Fe}^{\cdots} \cdot$ betrifft, so ist

$$
x \geqq 0 \text {, je nachdem } 3 a-c \geqq 0 \text { ist. }
$$

Bezeichnet man die Ionenkonzentrationen nach dem Zusatz von $d^{\prime}$ Ferrichlorid mit $\alpha a, \beta b, \gamma c, \delta d$ und $\varepsilon e$, so erhält man leicht die Beziehung :

$$
\alpha: \beta: \gamma: \delta=1: \varepsilon: \varepsilon^{2}: \varepsilon^{3} .
$$

Im vorliegenden Falle ist $\varepsilon>1$, da $3 x+2 y+z>0$ ist. Wird also die Ferrichloridlösung konzentrierter, so werden die komplexeren Ionen viel schneller in ihrer Konzentration zunehmen als die weniger komplexeren und umgekehrt. Aus dieser Erwägung ist ersichtlich, dass die Beziehungen $3 a-c\rangle,=,<0$ im Verlaufe der Konzentrationszunahme der Reihe nach befriedigt werden. $\mathrm{Fe}^{\cdots}$ ' wird also beim ständigen Zusatz von Ferrichlorid zum reinen Wasser anfänglich zunehmen $(3 a-c>0)$ und dann eine maximale Konzentration erreichen $(3 a-c=0)$, um dann abzunehmen zu beginnen $(3 a-c<0)$.

Im nächsten Falle wollen wir das Ferriion allein von der Lösung wegnehmen und dessen Einfluss studieren. Wegnahme des Ferriions allein geschieht derart, dass man von der Lösung das Ferrichlorid wegnimmt und durch Zusatz einer entsprechenden Menge Chlornatrium den Verlust an Chlorion kompensiert. Chlornatrium wird hierbei als vollständig dissoziierbar angenommen.

Die Konzentrationen der Ionen vor und nach der Wegnahme der $a^{\prime}$ Menge von $\mathrm{Fe}^{\cdots}$ sind :

Vor der Wegnahme Nach der Wegnahme

$\begin{array}{lll}\mathrm{Fe}^{\cdots} & a & a+x+y+z-a^{\prime} \\ \mathrm{FeCl}^{*} & b & b-x \\ \mathrm{FeCl}_{2} & c & c-y \\ \mathrm{FeCl}_{3} & d & d-z \\ \mathrm{Cl}^{\prime} & e+e^{\prime} & e+e^{\prime}+x+2 y+3 z,\end{array}$


wobei $e^{\prime}$ eine vorher im Überschuss zugesetzte Chlorionenmenge bezeichnet.

Ist $a^{\prime}$ sehr klein, so kann man die Gleichgewichtsbedingungen folgenderweise schreiben :

$$
\begin{array}{r}
\frac{x+y+z-a^{\prime}}{a}+\frac{x+2 y+3 z}{e+e^{\prime}}+\frac{x}{b}=0, \\
-\frac{x}{b}+\frac{x+2 y+3 z}{e+e^{\prime}}+\frac{y}{c}=0, \\
-\frac{y}{c}+\frac{x+2 y+3 z}{e+e^{\prime}}+\frac{z}{d}=0,
\end{array}
$$

woraus man erhält

$$
\begin{aligned}
& x=\frac{a^{\prime}}{a} \cdot \frac{2 c+6 d+e+e^{\prime}}{c d\left(e+e^{\prime}\right)} \cdot \frac{1}{d}, \\
& y=\frac{a^{\prime}}{a} \cdot \frac{-b+3 d+e+e^{\prime}}{b d\left(e+e^{\prime}\right)} \cdot \frac{1}{4}=\frac{a^{\prime}}{a} \cdot \frac{3 a+b+c+3 d+e^{\prime}}{b d\left(e+e^{\prime}\right)} \cdot \frac{1}{\Delta}, \\
& z=\frac{a^{\prime}}{a} \cdot \frac{e+e^{\prime}-2 b-2 c}{b c\left(e+e^{\prime}\right)} \cdot \frac{1}{d}=\frac{a^{\prime}}{a} \cdot \frac{3 a-c+e^{\prime}}{b c\left(e+e^{\prime}\right)} \cdot \frac{1}{\Delta},
\end{aligned}
$$

wobei $\Delta$ gleich wie im vorigen Falle ist, $e$ muss aber hier in $e+e^{\prime}$ umgeschrieben werden.

Man erhält ferner

$$
\begin{aligned}
& x+2 y+3 z=\frac{a^{\prime}}{a} \cdot \frac{b+2 c+3 d}{b c d} \cdot \frac{1}{\Delta}, \\
& x+y+z-a^{\prime}=-\frac{a x}{b}-\frac{a^{\prime}(b+2 c+3 d)}{b c d\left(e+e^{\prime}\right)} \cdot \frac{1}{\Delta} .
\end{aligned}
$$

Da, wie ersichtlich, $x, y$, und $x+2 y+3 z$ alle positiv und $x+y+z-a^{\prime}$ negativ ist, so folgt, dass durch Wegnahme des Ferriions $\mathrm{Fe}^{\cdots}{ }^{\cdots}, \mathrm{FeCl}^{*}$ und $\mathrm{FeCl}_{2}$ stets abnehmen, während $\mathrm{Cl}^{\prime}$ stets zunimmt. Was $\mathrm{FeCl}_{3}$ betrifft, so ist

$$
z \gtreqless 0, \text { je nachdem } 3 a+e^{\prime}-c \gtreqless 0 \text { ist. }
$$

Durch Wegnahme des Ferriions nimmt also $\mathrm{FeCl}_{3}$ bei hoher Konzentration zu; da aber $e^{\prime}$ bei fortwährender Wegnahme des Ferriions 
zunimmt, so muss bald $3 a+e^{\prime}-c \equiv 0$ werden, d. h. $\mathrm{FeCl}_{3}$ hierbei abzunehmen beginnen. Bei niedriger Konzentration oder in Gegenwart von genügend grossem Überschuss von Chlorion nimmt $\mathrm{FeCl}_{3}$ stets ab. Diese notwendige überschüssige Chlorionenmenge übertrifft schon die hinreichende, wenn sie einem Drittel der der Ferriionenmenge entsprechenden Chlorionenmenge gleich, d. h. $e^{\prime}=a+b+c+d$ ist, wobei $3 a+e^{\prime}-c=4 a+b+d$ offenbar immer positiv ist.

Schliesslich wollen wir den Einfluss von Zusatz des Chlorions allein studieren. Die Konzentration der Ionen vor und nach dem Chlorionzusatz $e^{\prime}$ sind :

$$
\text { Vor dem Zusatz Nach dem Zusatz }
$$

$\begin{array}{lll}\mathrm{Fe}^{\cdots} & a & a-x-y-z \\ \mathrm{FeCl}^{*} & b & b+x \\ \mathrm{FeCl}_{2} & c & c+y \\ \mathrm{FeCl}_{3} & d & d+z \\ \mathrm{Cl}^{\prime} & e & e+e^{\prime}-x-2 y-3 z,\end{array}$

wobei $e=3 a+2 b+c+e^{\prime \prime}$ ist, $\left(e^{\prime \prime}:\right.$ die vorher überschüssig

Falls $e^{\prime}$ sehr klein ist, so erhält man ähnlicherweise folgende drei Gleichungen :

$$
\begin{array}{r}
\frac{-x-y-z}{a}+\frac{e^{\prime}-x-2 y-3 z}{e}-\frac{x}{b}=0, \\
\frac{x}{b}+\frac{e^{\prime}-x-2 y-3 z}{e}-\frac{y}{c}=0, \\
\frac{y}{c}+\frac{e^{\prime}-x-2 y-3 z}{e}-\frac{z}{d}=0,
\end{array}
$$

woraus man erhält

$$
\begin{aligned}
& x=\frac{e^{\prime}}{e} \cdot \frac{a-c-2 d}{a c d} \cdot \frac{1}{\Delta}, \\
& y=\frac{e^{\prime}}{e} \cdot \frac{2 a+b-d}{a b d} \cdot \frac{1}{\Delta}, \\
& z=\frac{e^{\prime}}{e} \cdot \frac{3 a+2 b+c}{a b c} \cdot \frac{1}{\Delta},
\end{aligned}
$$


wobei $\Delta$ gleich wie oben ist.

Ferner gewinnt man

$$
\begin{aligned}
& x+y+z=\frac{e^{\prime}}{e} \cdot \frac{b+2 c+3 d}{b c d} \cdot \frac{1}{4}, \\
& e^{\prime}-x-2 y-3 z=\frac{e^{\prime}(a+b+c+d)}{a b c d} \cdot \frac{1}{\Delta} .
\end{aligned}
$$

$\mathrm{Da}$, wie ersichtlich, $z, e^{\prime}-x-2 y-3 z$ und $x+y+z$ positiv sind, so nehmen durch Chlorionzusat? $\mathrm{FeCl}_{3}$ und $\mathrm{Cl}^{\prime}$ stets zu und $\mathrm{Fe}^{\cdots}$ ab. Was $\mathrm{FeCl}^{\prime}$ " und $\mathrm{FeCl}_{2}$, betrifft, so haben wir

$x \gtrless 0$, je nachdem $a-c-2 d \gtrless 0$,

und $\quad y \gtrless 0$, je nachdem $2 a+b-d \gtrless 0$ ist.

Da $\quad 2 a+b-d=(a-c-2 d)+(a+b+c+d)$ ist, so bleibt die Beziehung $2 a+b-d>0$ bestehen sofern als $a-c-2 d>0,=0$ und noch etwas weiter. Wie oben gesehen, haben wir die Beziehung

$$
\alpha: \beta: \gamma: \delta=1: \varepsilon: \varepsilon^{2}: \varepsilon^{3}
$$

und da $\varepsilon>1$ ist, so werden $c$ und $d$ bei fortwährendem Chlorionzusatz vor $a$ und $b$ vorzuherrschen beginnen

Wir nehmen an, dass die anfängliche Ferrichloridlösung so verdünnt ist, dass die Bedingung $a-c-2 d>0$ bestehen kann. Setzt man zur Lösung ständigerweise Chlorion hinzu, so wird zu Anfang $\mathrm{Fe}^{\cdots \cdots}$ abund $\mathrm{FeCl}^{*}, \mathrm{FeCl}^{\circ}$ und $\mathrm{FeCl}_{3}$ zunehmen. Aber bald wird ein Punkt kommen, wobei $a-c-2 d=0$ wird. Von diesem Punkt an beginnt $\mathrm{FeCl}^{*}$ abzunehmen, während $\mathrm{FeCl}_{2}$ und $\mathrm{FeCl}_{3}$ noch ihre Zunahme fortsetzen, bis $2 a+b-d=0$ wird. Von da ab nehmen sowohl $\mathrm{FeCl}^{*}$ als auch $\mathrm{FeCl}_{2} \mathrm{ab}$, bis schliesslich fast alle Ferriionen in $\mathrm{FeCl}_{3}$ übergegangen sind.

Im folgenden wollen wir die Konzentrationsänderung der Ionen durch Chlorionzusatz etwas quantitativer behandeln.

Durch Zusatz von vollständig dissoziierendem Chlorsalz sei die Chlorionenmenge ver- $p_{-}$-facht. Ergänzt man die dadurch herbeigeführte Verminderung von freiem Ferriion (siehe oben) durch Zusatz 
desselben, so erhält man folgende Beziehung:

und deshalb $\quad \beta=\varepsilon, \gamma=\varepsilon^{2}, \delta=\varepsilon^{3}$.

Betreffs der Chlorionenmenge besteht die Gleichung

$$
(e+b+2 c+3 d) p=\left(e+b+2 \varepsilon c+3 \varepsilon^{2} d\right) \varepsilon .
$$

Da aber $\varepsilon>1$ ist, so muss

sein. Daraus folgt

$$
\varepsilon<p
$$

$$
\beta<p, \quad \gamma<p^{2}, \quad \delta<p^{3} .
$$

Entzieht man nun der Lösung die nachher zugesetzte Menge Ferriion, so werden $\beta$ und $\gamma$ abnehmen und auch so $\delta$, vorausgesetzt, dass die Beziehung $3 a+e^{\prime}-c>0$ besteht (siehe oben). Ver-p-facht man also die Chlorionenmenge, so erhalten wir die Beziehungen

$$
\beta<p, \quad \gamma<p^{2}, \quad \delta<p^{3},
$$

vorausgesetzt, dass die Lösung eine genügend überschüssige Chlorionenmenge enthält.

Ist die Lösung sehr verdünnt und das Ferrichlorid fast vollständig dissoziiert ist (ohne Hydrolyse), so wird

$$
\varepsilon \div p
$$

vorausgesetzt, dass $p-1$ sehr klein ist, denn dann $b, \beta b, c, \gamma c, d$ und $\delta d$ können gegen $a$ und $e$ vernachlässigt werden. In diesem Grenzfalle erhält man also

$$
\beta \doteqdot p, \gamma \doteqdot p^{2}, \delta \doteqdot p^{3} .
$$

Falls andererseits die Lösung sehr konzentriert ist oder einen sehr grossen Überschuss von Chlorion enthält, so wird $\delta$ fast gleich eins, denn $\mathrm{FeCl}_{3}$ kann nunmehr durch Zusatz von Chlorion nur sehr wenig zunehmen. $\beta$ und $\gamma$ werden, wie oben erwähnt, von einem Punkt an durch Zusatz von Chlorion abnehmen. Man dürfte also folgende Grenzen von $\beta, \gamma$ und $\delta$ als geltend ansehen:

$$
0<\beta<p, \quad 0<\gamma<p^{2}, \quad 1<\delta<p^{3} .
$$

Eine nähere quantitative Beziehung wäre schwer zu erhalten. 
376 N. Sasaki, Uber die Diessoziation Mehratomiger Salze.

\section{Zusammenfassung.}

Es wurde am Beispiel von Ferrichloridlösung mathematisch untersucht, wie die Konzentration des Moleküls und seiner Dissoziationsprodukte aller Stufen von der Gesamt-, von der Kationen- und von der Anionenkenzentration abhängen.

Es ist mir eine angenehme Pflicht, meinem hochverehrten Lehrer Herrn Prof. Dr. M. Chikashige für seine ständige Unterstützung für meine Arbeit an dieser Stelle meinen herzlichsten Dank auszusprechen. 